\title{
Correction to: Histological study of the thin membranous dense connective tissue around the middle and lower thoracic esophagus, caudal to the bifurcation of the trachea
}

\author{
Yutaka Tokairin ${ }^{1,2}$ (D) Kagami Nagai ${ }^{2} \cdot$ Yudai Kawamura $^{1,2} \cdot$ Yasuaki Nakajima $^{2} \cdot$ Kenro Kawada $^{2} \cdot$ Akihiro Hoshino $^{2}$. \\ Takuya Okada $^{2}$. Satoru Muro ${ }^{3} \cdot$ Keiichi Akita ${ }^{3} \cdot$ Yusuke Kinugasa ${ }^{2}$
}

Published online: 3 April 2021

(c) The Japanese Association for Thoracic Surgery 2021

\section{Correction to: General Thoracic and Cardiovascular Surgery https://doi.org/10.1007/s11748-021-01615-3}

The original version of this article unfortunately contained some mistakes. The errors were corrected in this correction as given below.

1. The first line of figure 2 legend should read as "A The histological findings near the bifurcation of the trachea of specimen 1 from cadaver 1 (EVG staining)." instead of "a The histological findings near the bifurcation of the trachea of specimen 1 from cadaver 1 (EVG staining)."

2. In page 5, the paragraphs "At the dorsal side of the esophagus, ..." and "On the ventral side of the esophagus, ..." should be in indent.

3. The first line of figure 5 legend should read as "An intraoperative photograph of the transhiatal approach ... " instead of "a An intra-operative photograph of the transhiatal approach ... “.

The original article can be found online at https://doi.org/10.1007/ s11748-021-01615-3.

Yutaka Tokairin

tokairin.srg1@ tmd.ac.jp

1 Department of Surgery, Toshima Hospital Tokyo Metropolitan Health and Hospitals Corporation, Tokyo 173-0015, Japan

2 Department of Gastrointestinal Surgery, Tokyo Medical and Dental University, 1-5-45 Yushima, Bunkyo-ku, Tokyo 113-8510, Japan

3 Department of Clinical Anatomy, Tokyo Medical and Dental University, 1-5-45 Yushima, Bunkyo-ku, Tokyo 113-8510, Japan
Publisher's Note Springer Nature remains neutral with regard to jurisdictional claims in published maps and institutional affiliations. 